\title{
Some New Insights Into Droplet Grouping Dynamics
}

\author{
Y. Geyari ${ }^{1}$, J.B. Greenberg ${ }^{* 1}$, A. Arad², D. Katoshevski², \\ V. Vaikuntanathan ${ }^{3}$, N. Roth ${ }^{3}$, B. Weigand ${ }^{3}$ \\ ${ }^{1}$ Aerospace Engineering, Technion - Israel Institute of Technology, Haifa, Israel \\ ${ }^{2}$ Dept. of Civil and Environmental Engineering, Ben-Gurion University of the Negev (BGU), \\ Beer-Sheva, Israel \\ ${ }^{3}$ Institute of Aerospace Thermodynamics (ITLR), University of Stuttgart, Stuttgart, Germany \\ *Corresponding Author email: aer9801@technion.ac.il

\section{Abstract} \\ Previous experimental studies of a vertical monodisperse non-evaporating isopropanol droplet \\ stream found that consecutive pairs of droplets drew close to one another, eventually \\ coalescing and continuing their fall. It is the phenomenon of the convening of pairs of \\ consecutive droplets in this configuration that is our fundamental paradigm for droplet grouping \\ in spray systems. \\ In the current work, we tackle the modelling aspects of the aforementioned configuration from \\ the vantage points of classical theoretical analysis. Our aim is to test the ability of previous \\ related research reported in the literature to ascertain the extent to which it is able to provide \\ a sound basis for predicting the experimental observations, thereby giving insight into the \\ mechanisms seemingly at play in droplet grouping dynamics. A model based on the Oseen \\ approximation is applied. The model is extended to consider the behaviour of pairs of droplets \\ having dissimilar radii and the case when the droplets are evaporating. Excellent agreement \\ with available experimental data was found.
}

\section{Keywords}

Droplet stream, grouping, droplet dynamics

\section{Introduction}

The efficiency of spray systems for various applications, from fuel combustion to precise surface coating, relies on the dynamics of liquid droplets, and among other things, on their tendency to form groups, a tendency that has an influence on their evaporation, the drag force applied to them, and on their final settling point. This predisposition has ramifications in energy and transportation systems, coating, as well as inhalation systems. Increased relevance is also assumed, in view of current trends of introducing biofuel blends in practical combustion systems.

In order to get a deeper understanding of droplet grouping a simple configuration was previously examined experimentally [1, 2]. An appropriately manipulated frequency was applied to a vertical isopropanol droplet stream generator thereby producing a monodisperse non-evaporating droplet stream. It was found that consecutive pairs of droplets drew close to one another, eventually coalescing and continuing their fall. It is the phenomenon of the convening of pairs of consecutive droplets in this configuration that is our fundamental paradigm for droplet grouping.

Two basic models for describing the subject of falling droplets/spheres are those of Stokes and Oseen. Stokes [3] developed the renowned expression for the drag force acting on a sphere moving through a viscous fluid (Stokes' law) for low Reynolds number. Oseen [4] suggested an improvement by accounting for the convection term in the momentum equation in an approximate way. He derived a drag force that essentially extends Stokes' law. 
Based on these fundamental works, further studies were conducted to better understand the motion of two (or more) falling spheres moving along their line-of-centre. These studies addressed the phenomenon whereby spherical objects moving together tend to change the surrounding flow field, thus affecting the drag force acting on each sphere. In some cases, there is even an attraction between the spheres, such that they eventually group together. Thus, Stimson and Jeffery [5] derived expressions for the drag forces acting on two spheres falling one above the other. The theory, which was based on Stokes' law, covered the case of two different-size spheres but did not address the attraction phenomenon, as it took the distance between the spheres to be constant.

Based on both Oseen's theory and the earlier work by Stimson and Jeffery [5], expressions for the drag forces acting on two falling spheres were obtained by Happel and Pfeffer [6]. These expressions were comprised of Stimson and Jeffery's drag force, which accounted for the general interaction between the falling spheres, and of an Oseen correction term, which accounted for the attraction phenomenon. Although the work of Happel and Pfeffer included experiments that validated their theoretical deliberations, it covered only the case of equalsized spheres (see, also, [7])

Many other studies proposed relatively complex mathematical models for simulating the motion of spheres in a group, or offered somewhat heavy numerical calculations for this purpose. For example, studies [8], [9] discuss the possible attraction between the spheres using complex models, but do not account for the case of different-sized spheres.

In the current work, rather than resorting to a time-consuming CFD solution, we propose a straightforward amendment to ideas expounded previously. In addition to considering the way in which two identical droplets falling along their line-of-centres draw closer to one another we also discuss the case when the two droplets are dissimilar in size. Finally, we examine the influence of droplet evaporation on the way in which the droplets draw closer together.

\section{The Physical Problem and Governing Equations}

In this section, the physical theory of the investigated problem will be discussed.

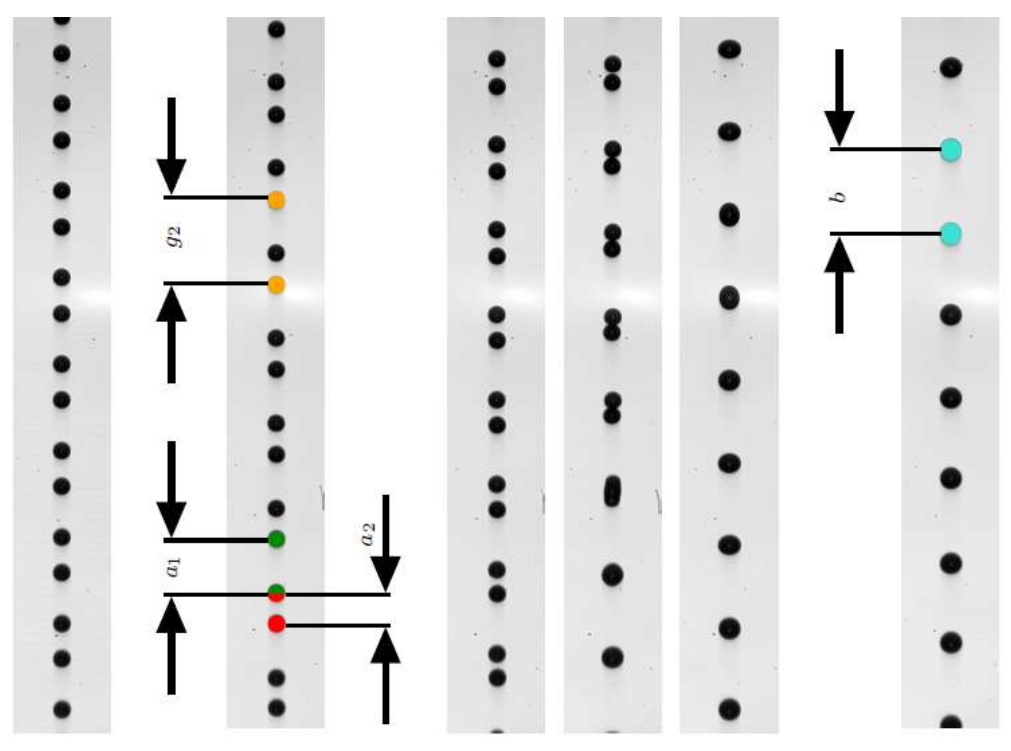

Figure 1: Typical sections of a stream of pairs of descending droplets; time increases from left to right - the notation defines various distances that will be referred to later $[1,2]$. 
We make use of the following assumptions: (a) Low Reynolds number based on the average droplet diameter and the terminal velocity, i.e. $\operatorname{Re}<1$, (b) droplets are modeled as semi-rigid spheres, in the sense that they always maintain a perfect spherical form, although they may also evaporate over time, (c) detailed coalescence effects are neglected. Once two droplets touch, they become one larger spherical droplet within an infinitesimally short time duration. The governing equations of motion for two droplets are:

$\left.m_{\text {Top }} \frac{d u}{d t}\right|_{\text {Top }}=\left.F_{d}\right|_{\text {Top }}+\left.F_{b}\right|_{\text {Top }}-\left.F_{g}\right|_{\text {Top }}$

$\left.m_{\text {Bottom }} \frac{d u}{d t}\right|_{\text {Bottom }}=\left.F_{d}\right|_{\text {Bottom }}+\left.F_{b}\right|_{\text {Bottom }}-\left.F_{g}\right|_{\text {Bottom }}$

subject to initial conditions:

$u_{\text {Top }}(0)=u_{0}$

$u_{\text {Bottom }}(0)=u_{0}+\Delta u$

where $m$ denotes mass of the droplet with the suffices self-evidently referring to the top and bottom droplets, $u$ is the velocity, $\Delta u$ is the increment in the velocity of the lower droplet when the upper droplet is initially released, and the forces $F$ acting on the upper and lower droplets are the drag forces, buoyancy forces and gravity (denoted by suffices $d, b$ and $g$ ), respectively. If the droplets are evaporating the following equation expressing the $d^{2}$-law is appended:

$[d(t)]^{2}=\left[d\left(t_{0}\right)\right]^{2}-K t$

where $d$ is the droplet diameter and $K$ the evaporation coefficient.

As we are mainly interested in the way in which the distance between the droplets $\left(a_{2}\right)$ behaves as they draw close to one another we subtract Eqs.(1) and (2) and obtain the equations:

$\frac{d a_{2}}{d t}=u_{T 0 P}-u_{B o t t o m}$

$\frac{d^{2} a_{2}}{d t^{2}}=\frac{F_{d, T o p}}{m_{\text {Top }}}-\frac{F_{d, \text { Bottom }}}{m_{\text {Bottom }}}$

subject to:

$a_{2}(0)=l$

$\frac{d a_{2}(0)}{d t}=-\Delta u$

where $l$ is the distance already travelled by the bottom droplet at the instant when the top droplet begins to move. We consider the drag forces acting on the top and bottom droplets to be comprised of a basic drag force and, following [6], an extra force experienced by the bottom droplet. The basic drag force will be computed using the formulae derived by Stimson and Jeffery [5]:

$F_{\text {base } 1}=-\frac{\mu_{f} \pi 2 \sqrt{2}}{a} \sum_{n=1}^{\infty}(2 n+1)\left(A_{n}+B_{n}+C_{n}+D_{n}\right)$

$F_{\text {base } 2}=-\frac{\mu_{f} \pi 2 \sqrt{2}}{a} \sum_{n=1}^{\infty}(2 n+1)\left(A_{n}-B_{n}+C_{n}-D_{n}\right)$

with the suffices base 1 and base 2 referring to the top and bottom droplets with radii $r_{1}, r_{2}$ ,respectively, in which the coefficients $A_{n}, B_{n}, C_{n}$ and $D_{n}$ are complicated expressions of hyperbolic and geometric functions involving the radii of the two droplets and the distance between them and $a$ is a constant chosen so that the droplets can have any radii and any 
centre distance greater than the sum of their radii. In fact, although Stimson and Jeffery [5] presented the aforementioned expressions, they only made use of a reduced form, which results from assuming the two droplets are identical in size. We retain the full expressions for generality and future reference. The extra force experienced by the bottom droplet, unlike that of [6], is due to Proudman and Pearson [10] who gave an improved Stokes' solution in the neighborhood of single sphere and an improved Oseen's solution at infinity, matching the two solutions in a common region of validity. Hence, the form of the two forces on the RHS of Eq. (7) is:

$$
\left(\left.F_{d}\right|_{\text {Top }},\left.F_{d}\right|_{\text {Bottom }}\right)=\left(F_{\text {basel }}, F_{\text {base } 2}-6 \pi \mu_{f} r_{\text {Bottom }} U\left(\frac{3}{8} \operatorname{Re}+\frac{9}{40} \operatorname{Re} e^{2} \ln R e\right)\right)
$$

In these last three equations $\mu_{f}$ is the viscosity of the fluid environment and $U$ is the average terminal velocity. Note that the last term on the RHS of Eq.(12) involving the In term is the more accurate improvement on the formula used by Happel and Pfeffer[6] for the drag on the bottom droplet. This accounts for slowing of the bottom droplet due to inertial effects. Eqs.(6) and (7) are applied to multiple pairs of droplets inserted in the vertical stream. The governing equations were solved using a standard MATLAB ODE solver.

\section{Results and Discussion}

The results to be discussed were based on use of the data listed in Table 1. We assume that the droplets are equal in size and non-evaporating. We compare the prediction of the theory with measured data of [1, 2]. We begin using Happel and Pfeffer's cruder model [6] for which the term $(9 / 40) R e^{2} \ln R e$ is absent in Eq.(12). In Figure 2 we plot the distance between two consecutive droplets $\left(a_{2}\right)$ and the distance between two consecutive pairs of droplets $\left(a_{1}\right)$, as functions of time. For both $a_{1}$ and $a_{2}$ fairly satisfactory qualitatively similar behaviour is obtained. A more detailed comparison is provided in Figure 3. In this figure, following notation in Fig. 1, the symbol $b$ signifies the distance between two consecutive pairs of droplets after each pair has coalesced, whereas the symbol $g_{2}$ stands for the distance between the bottom droplets in each consecutive pair, long before coalescence takes place, at an early stage of the motion. We did not account for the complex process of coalescence. Rather, instantaneous coalescence was assumed upon the two droplets in a pair coming into contact with one another. This is not unreasonable, as the experiments indicate a typical time of coalescence of the order of $10^{-4} \mathrm{~s}$ whereas a characteristic time for the motion until a pair of droplets convene is one order of magnitude greater. The size of the resulting single coalesced droplet can be straightforwardly found using conservation of mass. The various distances appearing here are plotted as a function of distance from the orifice of the apparatus producing the droplets. There is overall qualitative agreement for those distances relevant to the precoalescence stage. For the post-coalescence period the agreement is less satisfactory, although correct orders of magnitude are predicted.

We now allow the droplets to evaporate according to Eq.(5). Note that, in the experiments, it was assumed that the droplets are at a constant temperature precluding their evaporation. Figure 4 shows the effect of permitting droplet evaporation during the fall of the droplets. We observe that the effect of allowing droplet evaporation is deleterious, as the curve of the predicted distance between the droplets distances itself further away from the experimental results than the equivalent curve obtained without droplet evaporation. This would seem to 
Table 1 - Data used for numerical solution.

\begin{tabular}{c|ccc}
\hline & Parameter & Value & Units \\
\hline Droplets & $d$ & 112 & $\mu \mathrm{m}$ \\
(isopropanol) & $\rho$ & 784 & {$\left[\mathrm{~kg} \cdot \mathrm{m}^{-3}\right]$} \\
& $K$ & 783 & $\mu \mathrm{m}^{2} \cdot \mathrm{s}^{-1}$ \\
Medium & $\rho_{f}$ & 1.196 & {$\left[\mathrm{~kg} \cdot \mathrm{m}^{-3}\right]$} \\
Fluid (air) & $\mu_{f}$ & 1.83 & {$[\mathrm{~kg}(\mathrm{~m}$} \\
& & $\cdot 10^{-5}$ & $\left.\cdot \mathrm{s})^{-1}\right]$ \\
Initial & $u\left(t_{0}\right)$ & -14.9 & {$\left[\mathrm{~m} \cdot \mathrm{s}^{-1}\right]$} \\
Condition & & &
\end{tabular}

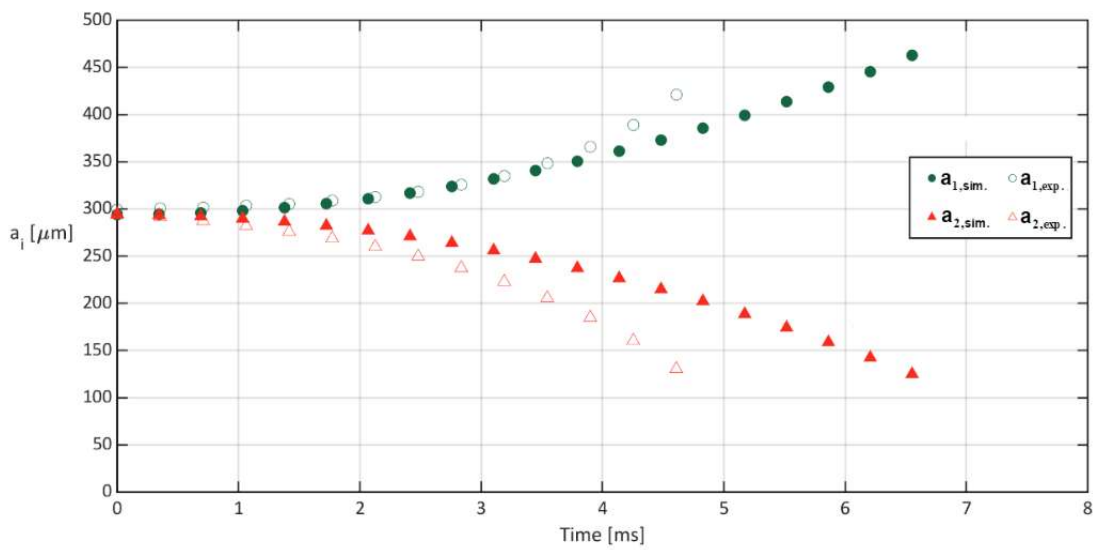

Figure 2: Distance between two consecutive droplets $\left(a_{2}\right)$ and between two consecutive pairs of droplets $\left(a_{1}\right)$ as a function of time; comparison between measured and predicted results using Happel and Pfeffer [6] theory.

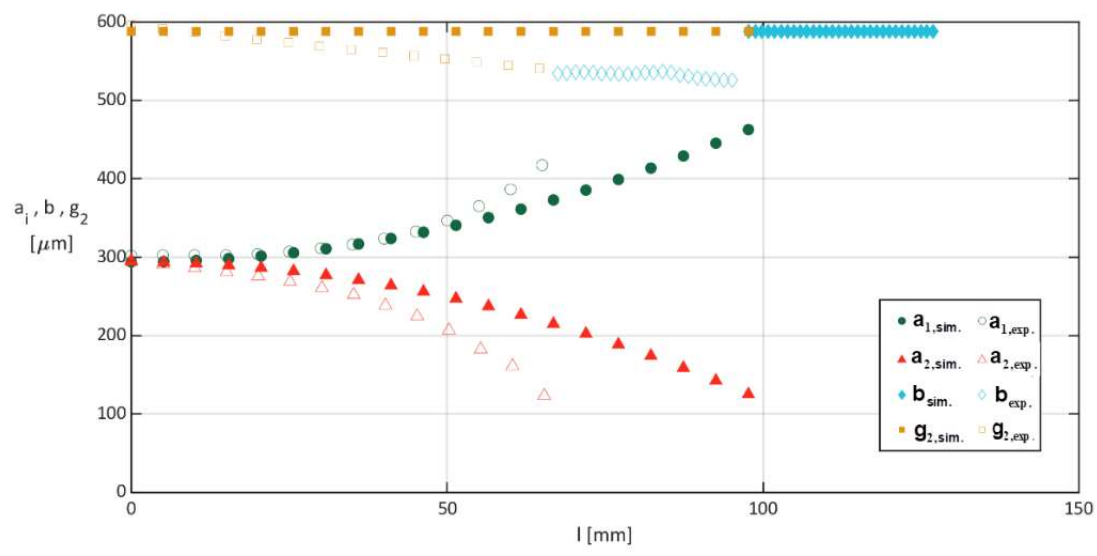

Figure 3: Various distances of droplets in pairs as function of distance to the orifice (see text for definition of distances)

support the correctness of the aforementioned constant temperature assumption of the experiments.

In contrast to Figure 3, results obtained using the refined theory of Proudman and Pearson[10] as included in Eq.(12) are shown in Figure 5. For $a_{1}$ the comparison with experiment is, once again only qualitatively similar. However, the match between the distance between two droplets and the experimental measurements is quite remarkable. What is surprising is that whereas the theory is predicated on a Reynolds number less than unity, the experiments were 


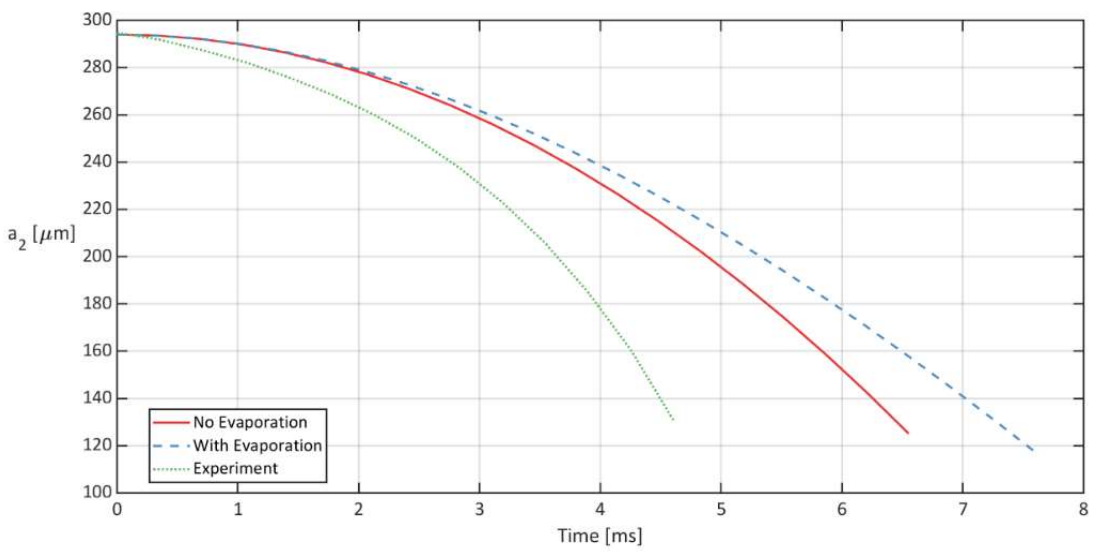

Figure 4: Effect of allowing the droplets to evaporate on the distance between two consecutive droplets $\left(a_{2}\right)$

performed with a reported Reynolds number of the order of 100. Figure 6 summarizes a comparison between experiment, the drag force used by Happel and Pfeffer [6] and the improved drag force for the bottom droplet. The vast improvement delivered by the latter is clearly visible, seemingly indicating that matching the Stokes solution near a single sphere and the Oseen solution at infinity in a common region of validity captures essential features of the flow field in the vicinity of the pair of droplets.

Finally, in Figure 7, Figure 3 is re-drawn with the improved drag force on the bottom droplet. Despite the improvement in the pre-coalescence curves, the post-coalescence distances remain generally unchanged.

The way in which the droplet motion is considered here can be termed a far-field view. This does not account for any fluid motion within the droplets nor any fluid motion between the droplets, as well as any influence of the droplets on the surroundings into which they are injected. Included in the latter is the way the flow field in between pairs of droplets impacts on their behaviour. Work by Kotsev [11] makes a step in this direction in simulations for a problem allied to one considered here carried out using the commercial COMSOL code. However, flow over a single pair of solid spheres fixed relative to one another is computed. The results provide an insight into the way the flow field around and between spheres varies as a function of the distance between the spheres centres and the Reynolds number. The proliferation of vortices that are formed as the Reynolds number increases from 10 to 200 will drastically effect the drag forces on the spheres. The top sphere faces a reduced drag compared to the bottom one, decreasing rapidly with $\mathrm{Re}$, and becoming negative for $\mathrm{Re}=200$ implying a weak attractive effect between the spheres. In general terms, this is in consonance with the ideas of [6] for low Reynolds numbers. However, since solid spheres are studied in [11], motion within liquid droplets is excluded. A closer view, which includes all the aforementioned intradroplet, inter-droplet and droplet-surrounding fluid effects, will necessitate more comprehensive CFD efforts. These will undoubtedly reflect the physics more accurately, but at the cost of high-powered computing facility simulations, which are not without their own limitations. In view of the relatively simple framework we have used, the question remains why the application of the classical theories applied in the current work manages to replicate the experimental results for the time variation of the distance between two consecutive droplets so well. 


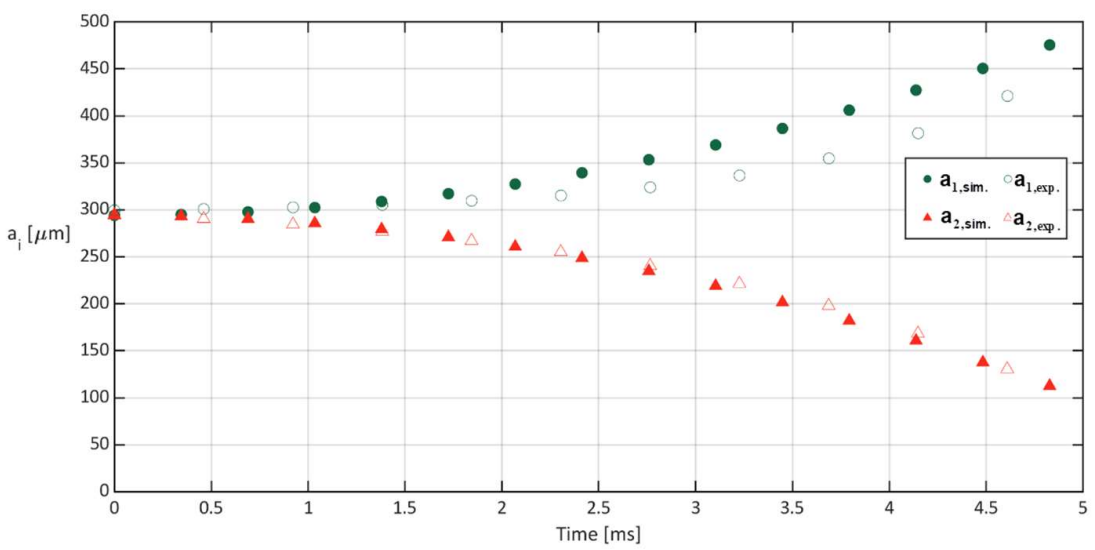

Figure 5: Distance between two consecutive droplets $\left(a_{2}\right)$ and between two consecutive pairs of droplets $\left(a_{1}\right)$ as a function of time; comparison between measured and predicted results using improved drag force term (Eq.(12))

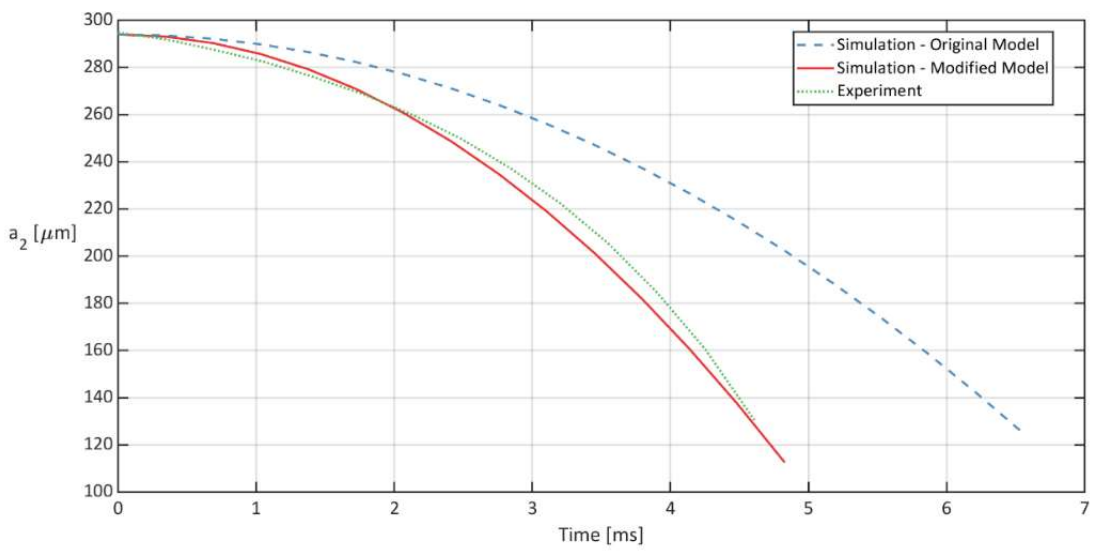

Figure 6: Distance between two consecutive droplets $\left(a_{2}\right)$ as a function of time; comparison between experimental data and predictions of original theory and improved theory.

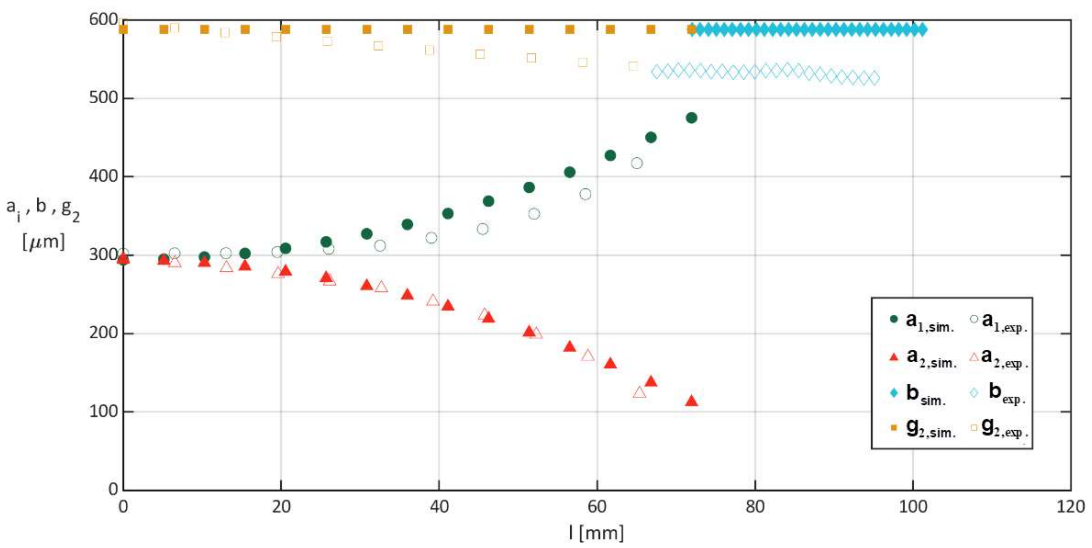

Figure 7: Various distances of droplets in pairs as function of distance to the orifice (see text for definition of distances) using the improved drag force on the bottom droplet (Eq.(12). 


\section{Conclusions}

The dynamics of the vertical motion of pairs of droplets and their grouping was examined using classical theory from the literature. An improved drag force acting on the bottom droplet of each pair was suggested, based on the more comprehensive analysis of Proudman and Pearson [10]. It was found to enable excellent replication of the experimental measurements for the evolution of the distance between a pair of droplets. This agreement is surprising, given the large disparity between the Reynolds number of the experiments and the Reynolds number less than unity upon which the classical expressions were based. Since the standard drag force acting on a droplet requires integrating the stress tensor on the body surface it is hoped that data from detailed CFD calculations will shed light on the success of the current simplified model.

\section{Acknowledgments}

This study was performed with the financial support from the Deutsche Forschungsgemeinschaft (DFG) through the project 'Investigation of droplet motion and grouping' (project number 409029509).

\section{References}

[1] Roth, N., Weigand, B., Katoshevski, D. \& Greenberg, J. B., 2015, "Basic Studies on Grouping Effects in Droplet Streams". Droplet Impact Phenomena and Spray Investigations (DIPSI) Workshop, Bergamo, Italy.

[2] Roth, N., Gomaa, H., Livne, A., Katoshevski, D., Weigand, B., 2017, ILASS, 28th Conference on Liquid Atomization and Spray Systems, Valencia, Spain.

[3] Stokes, G. G., 1851. Transactions of the Cambridge Philosophical Society, 9, pp.8-10.

[4] Oseen, C. W., 1927, "Hydrodynamik". Akademische Verlagsgesellschaft, Leipzig, Germany.

[5] Stimson, M. and Jeffery, G. B., 1926, Proceedings of the Royal Society Series A, 111(757), pp. 110-116.

[6] Happel, J. \& Pfeffer, R., 1960, AIChE Journal, 6(1), pp. 129-133.

[7] Happel, J. \& Brenner, H., 1981, "Low Reynolds Number Hydrodynamics". Martinus Nijhoff Publishers, Leiden, Netherlands.

[8] Bot, E. T. G., Hulsen, M. A. \& van den Brule, B. H. A. A., 1998, Journal of Non-Newtonian Fluid Mechanics, 79, pp. 191-212.

[9] Rosenstein, Y., Leshansky, A. M., 2012, European Journal of Mechanics B/Fluids, 31, pp. 71-79.

[10] Proudman, I. and Pearson, J. R. A., 1957, Journal of Fluid Mechanics, 2(3), pp. 237-262.

[11] Kotsev, T., 2018, MATEC Web of Conferences 145, 03008, https://doi.org/10.1051/matecconf/201814503008. 